\title{
Ambient interaction and situational influence: case studies in public sites.
}

\author{
HER, J.-J. and HAMLYN, J.
}




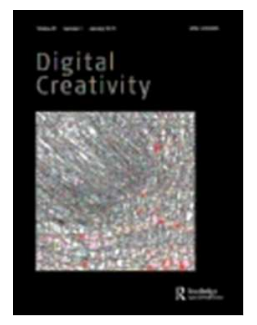

Ambient Interaction - Interactivity as a creative medium

\begin{tabular}{|r|l|}
\hline Journal: & Digital Creativity \\
\hline Manuscript ID: & Draft \\
\hline Manuscript Type: & Technical article \\
\hline Keywords: & Interactivity, Ambient Interaction, Interactive Art, Public Art \\
\hline \multicolumn{2}{|c}{} \\
\hline
\end{tabular}

\section{SCHOLARONE $^{\text {M }}$}

Manuscripts 


\title{
Ambient Interaction - Interactivity as a creative medium
}

\begin{abstract}
An audience's physical intervention is often considered to be instrumental in the field of interactive art. However artworks displayed in public spaces are often invested with diverse purposes, in which many do not directly involve audiences or audience members as key components for realising the intentions of the art. This raises the question of whether direct interaction with audiences is considered to be essential, in particular for those interactive artworks displayed in public venues outside conventional art spaces. This article introduces an important characteristic of interactivity in interactive art, through the largely unexplored concept of 'Ambient Interaction' in which artworks come to be embodied by environmental conditions and situational influences rather than exclusively through people's conscious and direct intervention. In such cases, it is argued, artists would benefit from a broader awareness of not only whom, but what their artworks will encounter and interact with.
\end{abstract}

\section{Introduction}

This article proposes the concept of 'Ambient Interaction'. Rather than directly associating interactivity with people, by interacting with environmental influences (e.g. light, temperature, sound, movements of passersby or other causal agents), artworks produce diverse (ambient) interactivities, through which aesthetic quality and intent are manifested and augmented/transformed. This concept initially appeared during several field observations of interactions between people and different interactive artworks displayed in freely accessible public places outwith conventional art spaces (including the façade of buildings, MRT stations and an airport). The concept gradually crystallized after comparing the original artworks' introductory statements and the ways in which people interacted with three interactive art installations on site. With the purpose of elaborating the concept of 'Ambient Interaction', it is inevitable that the two constant mutable terms 'Interactive Art' and 'Public Art' will be prominent. However, it is important to state that at this stage that the objective of this article is by no means to become entangled in debates with these already widely contested and discussed definitions, instead it is to highlight their foundations in order to discuss and elaborate the notion of 'Ambient Interaction'.

Over the past decade interactive technologies and devices have become increasingly accessible by artists and designers alike, this, to some extent, has encouraged the crafting and exhibition of various interactive artworks not only in art galleries and museums, but also in public spaces, the latter of which is currently burgeoning. However, when a media-based art installation enters the public domain it simultaneously encounters obstacles, among which two primary issues are frequently raised. Firstly, people in urban public places are often 
'involuntary audiences' (Senie cited by Knight 2008 p.28), as they usually have other goals in mind and have no explicit intention of engaging in an artistic experience. Boros (2012 p. xii) remarks that "art is in the public means it reaches more people more often, and it often reaches people who may not normally seek out art". Secondly, in public spaces it is inevitable that artworks will be confronted with other omnipresent media and digital devices/environments. In such cases people's attention can be significantly distracted, with many failed attempts at engaging potential audiences. Müller et al (2010) point out that: "many displays seem to fail to attract enough attention of passersby, simply vanishing in the cluster of things in public spaces that compete for attention". Moreover they also claim that "if public displays fail to attract enough audience attention however, they may not be used at all" (ibid).

The above two challenges have drawn substantial resources and efforts into the research of interactive public displays with the objective of capturing people's attention at appropriate times, provoking motivation, sustaining experiences and facilitating utilization of digital interfaces and/or further realising artistic intent (e.g. Müller ibid, Huang et al 2009, Petersen et al 2004, Brignull and Rogers 2003, Tikka et al 2011). Among these, many were Human Computer Interface (HCI) based studies, while the majority focused on usability and the effectiveness of navigating interfaces in order to assist users in obtaining information accurately and without frustrating delays. Sundar (2010) indicates that "HCI researchers have long demonstrated the powerful influence that modalities of interaction have on humans". This, however, may not agree with artists' interests and intentions as they often "make works that fail in every way imaginable intentionally" (Paulos 2007). Bialoskorski (2010) reinterpreted the argument of Höök et al (2003) stating that: "evaluating this kind of interactive systems is common in human-computer interaction (HCI), but it is not common to test interaction in art. This is because HCI evaluation strives to be objective, while in art it is all about the subjective opinion of a single observer"[sic]. Edmonds (2011) in his article Art, Interaction and Engagement writes: "the behaviour of the works is not intended to always be obvious, so that if you continuously try to force a response by waving it might result in a period of quiet". In such cases people may misunderstand, thinking instead that the artwork has malfunctioned, however it may sometimes be a part of an artist's strategy to disrupt conventional patterns of response and expectation. Artists might therefore be prepared to forego the engagement of some less attentive audience members in preference for the occasional viewer/participant who makes a deeper or more rewarding connection with the work. 
Nevertheless divergence between HCI and art research/creation may not be inevitable. Höök et al (2003) have argued that HCI methods can offer benefits which may improve the creation of interactive artworks. In addition, according to Ciolfi et al (2008) "in the past decade or so the field of $\mathrm{HCI}$ and interaction design has become less defined by an explicit work orientation regarding the design of technology, and is increasingly concerned with issues of fun, enjoyment and aesthetics." Edmonds (2010) also remarks that, "the knowledge of HCI and its methods can contribute to interactive art making." The discussion in this section is by no means intended to oppose the relevance of HCI to art research, but rather to highlight a phenomenon of the research with regards to interactive artworks, particularly concerning the interaction between artworks and people, which, to some extent, bypasses a vital aspect of the relationship between artworks and other ambient causal mechanisms and influences. This unwitting ignorance may result from the fact that the aesthetic quality of interactive art is commonly defined by its interaction between artworks and people. However when art steps into the public sphere it undergoes transformations on a number of levels simultaneously, with unanticipated consequences. A well-known example is Richard Serra's public sculpture 'Tilted Arc' (1981) which itself became a catalyst for positive aesthetic awareness, social interaction and the usability of Federal Plaza whilst also encountering substantial criticism and resistance that ultimately lead to its unanticipated removal from the site in 1989 (Horowitz 1996 and Hein 1996).

Based on in-depth literature reviews on interactive art, art in public spaces, along with field observations of interaction between interactive artworks, and people with their surroundings, the following sections will progressively dissect the concept of Ambient Interaction. It is expected that this concept will help to materialize the artistic characteristics of interactive art so as to enable more fruitful and diverse interactivity in public places.

\section{Widespread Recognition of Interactive Art}

A significant amount of literature emphasises as a key feature of interactive art; its association with people, and their mutual (direct and conscious) influences. Popper(2007 p.181) remarks that in interactive digital installations: "interactivity can be interpreted as the ability of the user to manipulate and affect one's experience of media directly, and to communicate with others through media". Bilda and Edmonds (2008) note that "interactive art invites the audience to engage through interaction and, in doing so, participate in the realization of the work itself". Moreover, Kim. T and Kim. K (2012) claim that "interactive art is not successful if the audience engages in no further interaction with the work". Heinrich (2012) also states that "in interactive art the onlooker has finally become a participant physically acting and reacting within the timely and spatial framework of an interactive art piece." There are a great number of references underlining the fact that a participant's physical intervention within the 
process of interactive art fulfills a crucial role in terms of the satisfactory realization of interactive artworks (e.g. Rokeby 1995, Sims 1997, Ascott 2001, Morse (Malloy 2003), Rogala 2005, Dezeuze 2010 et al).

In addition, many researchers and artists further assert that the real quality of interactive art is manifested when physical and intellectual aspects are fulfilled, for instance, Holmes (Ascott 1999 p.90) states: "the interactive art experience is one that blends together two individualized narratives. The first is the story of mastering the interface and the second is about uncovering the content that artists bring to the work". Kravagna (Dezeuze 2010 p.241) argues "interactivity goes beyond a purely perceptual proposition in that it allows for one or more reactions to affect the work - usually in a momentary, reversible and repeatable manner - in its appearance, but without fundamentally changing or co-determining its structure". Without people's physical participation, or in the face of inappropriate responses, artworks may often be deemed as having missed a vital piece of the jigsaw or even of failing outright (Knickmeyer and Mateas 2005, Edmonds et al 2004).

Regardless of whether the above arguments rest upon the interactive installation's function as an aid to facilitate interactivity - of which the participant's involvement is fundamental - the intervention and involvement of people is regarded as an indispensible element in the actual fulfillment of an interactive artwork. Indeed a great number of interactive art installations have been devised with the primary intention of interacting with people, and only through interaction with them can the artistic intent be accomplished (although it is recognized that other important aspects of the work may mitigate this deficiency). This conventional interpretation of interaction may be a product of widespread recognition and definition, which has frequently reflected both the research and creation of interactive art. Since very little literature has tapped into the realm of interactivity occurring between artworks and other causal mechanisms/environments, this prevalent attitude towards interactive art may have restricted an essential quality of interactivity as a creative medium. However when considering interactivity in a wider frame of reference, regarding it instead as a contemporary art medium, equivalent to other conventional forms that are utilized to explore and/or convey artistic intents, the possibility, variability and creative potential of interactive artworks can be dramatically expanded.

\section{Interactivity - A Creative Medium}

In 1977 Krueger coined a term; 'response is the medium' in his influential publication Responsive Environments. In this article he presented another significant concept: "interactive art is potentially a richly composable medium quite distinct from the concerns of sculpture, graphic art or music," since, as Lev Manovich (2001) writes: "the traditional concept of a medium emphasizes the physical properties of a particular material". In traditional art 
disciplines, artists often resort to art materials and static art forms as communicative media to embody their ideas. Interactive artists meanwhile present their ideas through physical but nonetheless immaterial interactions. As such the interactivity itself comes to figure prominently as a medium in its own right, just as paint serves as a medium for painting. According to Larsson (2011) interactivity as often presumed to be an intrinsic quality of the new medium. Sundar (2010) underlines; "interactivity as a medium feature comes in the form of different modalities of information dissemination". In addition, Edmonds (2010 p.263) states that "Interactive art is as valid as any other [art] form. In making it, the artist deals with the same issues and faces much the same challenges as in any other kind of art. However, each form and each medium has its own set of specific problems and this one is no exception."

Unlike traditional art forms, interactive art is characterized by its tangible interactivity rather than its material qualities; it is embodied as a composition or an integrated art presentation of mixed materials, technology, and media. Interactivity is therefore, in itself, hybrid in its unique attributes, thus it can be conceived as a compound creative medium in which a variety of materials, processes and forms combine. This "medium is the channel through which the content is shared" (Sundar ibid). The sharing and exchange process in this discussed context traverses the boundary of people and artworks, and is broadened to the interrelations among artworks, other non-human agents and environmental influences. Through the course of sharing and exchange, the meaning of art is manifested. Dewey (2005 p.298) proposes that: "A medium as distinct from raw material is always a mode of language and thus expression and communication." Müller et al (2006) claim "meaning occurs through the process of exchange, and interactivity itself is the very medium of the work." With the purpose of improving the creation of interactive artworks Höök et al (2003) conducted an interactive experiment and published the outcomes in Sense and sensibility: evaluation and interactive art. In this article they explained that "artists tend to think of their systems as a medium through which they can express their ideas to the user".

Of course, interactivity does not exist solely in interactive art, it is also utilized in other traditional and contemporary art forms. However, despite the fact that literature in different research contexts has attempted to arrive at a definitive explanation of the word 'interactivity', they often incur disagreement and contradiction (Down and McMillian 2000). On this subject Kravagna (Dezeuze 2010 p.241) notes that "the boundaries are permeable, and that rigid categories are not useful." It is therefore evident that using this term without defining it in a specific context can lead one into a quagmire of debate. Thus, to increase clarity here the aim of this article will be reiterated; the focus is on interactivity as an art medium: through interaction with causal agents and environmental influences, meaning is manifested as art. 


\section{Art of Interactivity in Public Spaces}

As previously mentioned, a prevalent notion of interactivity in the field of interactive art views people as the prime or even exclusive source of aesthetic interaction; in this respect it is only through direct interaction with people that interactive art can be fully appreciated. However, this widespread interpretation of interactivity may underestimate the possibilities of interactivity as a creative medium, furthermore it can undermine artworks, in particular those which have been exhibited in public places. When art enters such public spaces it often carries with it various artistic purposes, responsibilities, and/or functions. Upon those conditions a direct interaction with people may not be prioritized because drawing attention, eliciting curiosity, triggering motivation, and prolonging physical engagement with audiences may not be the artists' primary concern when conceiving of or creating a public artwork. Knight (2008 p.22) notes that many artworks "are completely absorbed into the surroundings they literally escape notice as art; they nudge at and whisper to us that we perceive their effects in subtle ways. Other works scream for our attention; unwilling to be mitigated by site or circumstance. They insist we pay them mind." The former type of public artworks merge into environments, quietly settling where they are installed, while undergoing interplay with their surroundings implicitly. Through modest interactivity, the features of the space are enlivened, the quality of a space is improved, and so the transformative potential of the art is realised. We suggest that the same principle of implicit interplay would prove valuable if taken into consideration when applying interactivity as an artistic component in the creation of public artworks.

When discussing 'Art in Public Places', Lacy (1995 p.21) points out that in the 1960s, "the ability of art to enhance public spaces such as plazas, parks, and corporate headquarters was quickly recognized as a way to revitalize inner cities." Hein (1996) states that "conventionally the term Public Art refers to a family of conditions including the object's origin, history, location, and social purpose." Hein (ibid) continues: "Yet today's public artworks still have a conceptual link with traditional art presentation". Despite the fact that recent decades have witnessed diverse new forms and approaches towards public art, a substantial quantity of public artworks, including interactive artworks, continue to employ traditional approaches and have been selected for public spaces/places with the purpose of reflecting craftsmanship, aesthetic quality, and the history of a specific location. For instance, Listening Post (Hasen and Rubin 2002) was "designed to convey the magnitude and diversity of online communication" and the artists' "goal is to distill the content and the structure of this collective communication and to present in ways that are accessible and compelling"(ibid). Without the unwitting and indirect interaction of anonymous participants, the project could not exist. Selwood (1995 p.124) states that "the users of a space are also its producers (Deutsche 1992:161); without their agency a place lies dormant, awaiting human interactions and interventions to shape it." The role of humans here equates with other environmental 
factors, and although there may not be direct involvement per se, without their intervention the art remains incomplete.

Due to its well-known identity and status as digital art, Listening Post is included here as an example of ambient interactivity, in particular its indirect interaction with humans for its artistic realization. We are aware, of course, that Listening Post was awarded a Golden Nica in the interactive art category of the 2004 Prix Ars Electronica and may therefore be regarded as a contentious case (Huhtamo 2004). Nevertheless, we argue that it represents is a highly pertinent example by revealing shifting attitudes not only towards the meaning of the term 'Interactive Art', but also towards 'Public Art' (Listening Post's sources of interaction issued from directly the internet; a context widely regarded as a new form of public space).

\section{Case Studies - The Three Interactive Artworks}

An initial intention for the field observation was to select suitable artworks for further examination of the analytical framework; a conceptual research tool for analysis of interactivity between participants and interactive artworks so as to facilitate artistic interaction and prompt meaningful interactive experience (Author 2011, 2013). The criteria for the artwork selection was:

\section{1: Computer-operated interactive art installations}

2: Real time multimedia effects in response to input from the participants

3: Output effects from the artworks perceivable on site

Through the course of artwork selection, the concept of Ambient Interaction emerged without prior expectation. With the aim of providing a condensed argument for Ambient Interaction, three (permanent) interactive art installations in Taiwan have been selected. These are located as follows: on the façade of an industrial building at Jungli city, in the Taipei MRT (Mass Rapid Transit) Nangang Exhibition Center Station and at the Taoyuan international airport, they are included in this section to delineate the concept.

Between Moving and Still (Figures 1-1 - 1-3) is a sound and light based interactive installation, it has been exhibited on the façade of Taipower Jungli, Zi Li electrical substation since 2010: a plain, gray, cubic contemporary functional building in the typical style of electrical substations in Taiwan. The sound-based interactive LED artwork was created with the purpose of reviving the space and enhancing the overall impression of the power substation. This artwork consists of 147 pieces of $2.8 \mathrm{~cm}$ handmade stained glass and LED lights. The interactive mechanism was designed to respond environmental sound and control the sequencing of LED lights (ie: the higher the volume the taller the illuminated strip). When differing levels of sound are detected, a dynamic and colorful LED display is triggered (Taiwan Public Art 2012). 


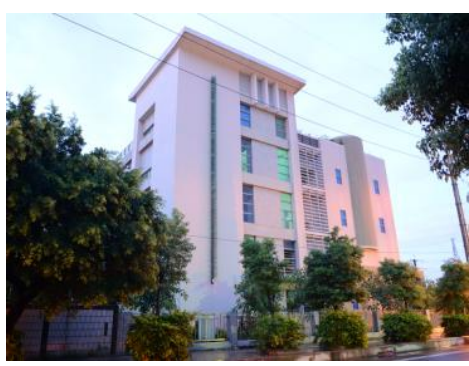

Figures 1-1

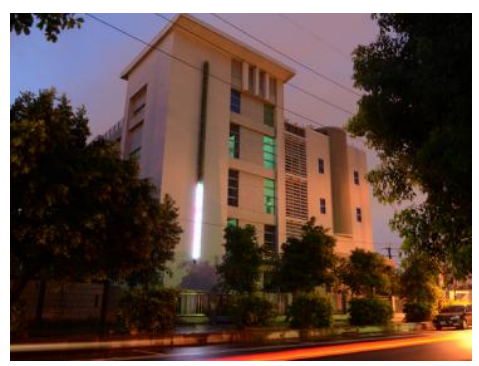

Figures 1-2

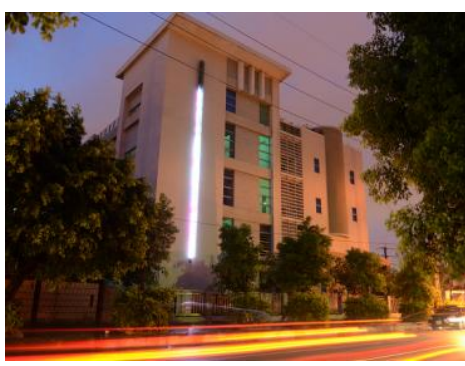

Figures 1-3

Fast or Slow (Figures 2-1 - 2-3) is also an LED based interactive artwork, it is displayed on the wall surfaces of a main corridor leading to the station concourse in Taipei MRT, Nangang Exhibition Center Station since 2011. This artwork is comprised of LED light bars, infrared sensors and aluminum plates. The LED light patterns and their moving speeds are influenced by passersby who walk at a predetermined distance and pace. The three modes of LED light performance are intended to symbolize different relationships between people, environments and societies. For instance, "When a passer-by walks along the wall on either side, the LED panels near him turns on and off, which represents his social participation" [sic] (Miura 2011). Additionally the static images of tree and water on the walls above and underneath the LED bars also contain artistic narratives; the overlaid multiple branches figuratively depict an intricate social system, while the water ripples are intended to suggest the fluidity of social relations (ibid).

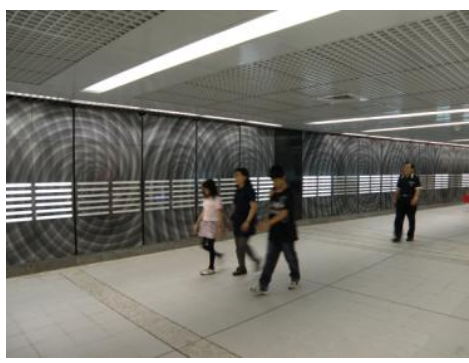

Figures 2-1

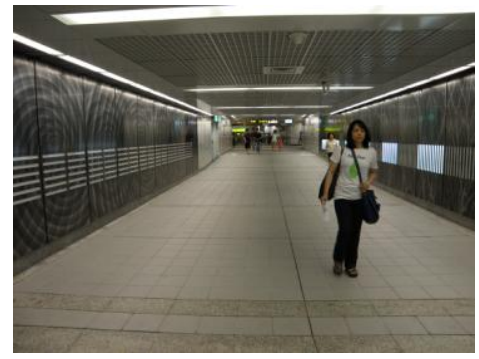

Figures 2-2

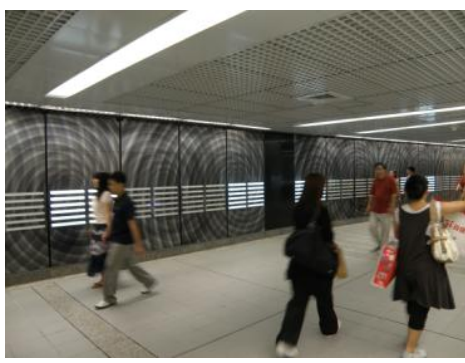

Figures 2-3

The Flower Clusters (Figure 3-1 - 3-3) has been exhibited at the Taoyuan international airport, terminal two since 2012. This art installation has separate input and output components, the input component being an LED screen with a digital camera mounted at its top edge. The output is incorporated into a stainless steel sculpture in the form of a blossoming flower with each petal comprised of a LED monitor. The input console is attached to the wall (see the red circle on Figure 3-1) at a participant approachable location, several meters away from the main flower sculpture. On most occasions the input console shows the introductory information for the artwork (JiunsView 2013), while the flower sculpture operates autonomously as a kaleidoscope, randomly displaying diverse, colorful patterns. Occasionally, the input console shows surrounding images, when that appears, any participating individuals 


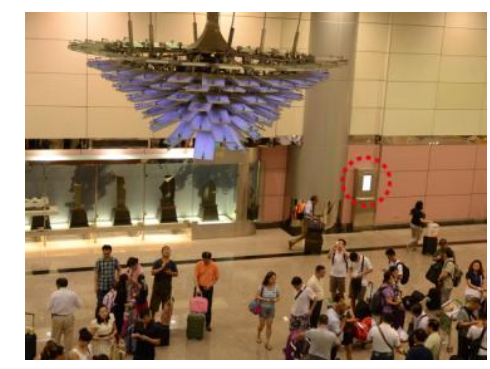

Figures 3-1

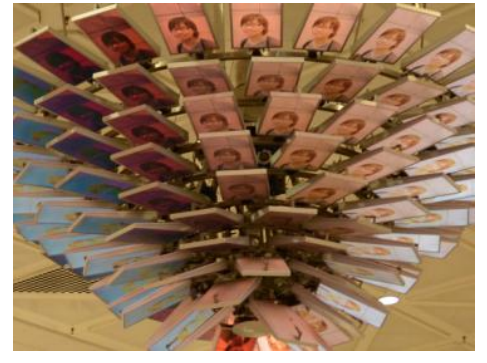

Figures 3-2

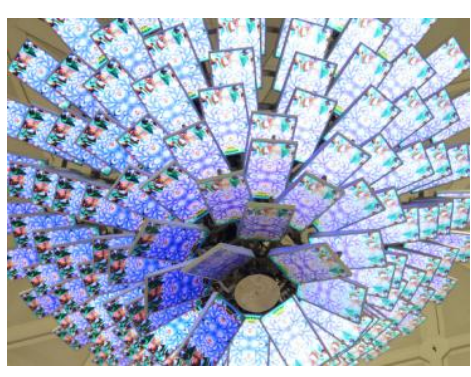

Figures 3-3

\section{Field Observations and Unstructured Interviews}

The concept of Ambient Interaction emerged during several field observations, which were conducted in urban, public places in Taiwan between June 2012 and January 2013, along with unstructured interviews, which were only carried out when people were glancing over or had stopped to watch the artworks. Regarding the arguments of interactivity as a creative medium, and art in public spheres often being endowed with diverse purposes, we attribute indirect or inadvertent interaction in this research context as having the potential to reveal unexpected and unforeseen qualities and associations that, instead of detracting from the work or undermining its intended use or meaning, contribute to its reinvention and/or reinvigoration. Indeed although a direct and conscious intervention from people can be important, it may not be necessary in terms of materializing artworks as it can be embodied through interaction with ambient conditions such as wind, light, sound, temperature and so forth. Miwon Kwon (2004 p.67) states that "public art would no longer be just an autonomous sculpture but would be in some kind of meaningful dialogue with, maybe even coincide with, the surrounding architecture and/or landscape."

Between Moving and Still is one such artwork, as it performs interactively based on the different magnitude of sounds from ambient sources, a louder sound drives higher LED light displays or vice versa (Figures 1-1 - 1-3). In the daytime it glimmers to its surroundings as the changes of LED light are barely visible. When evening arrives it comes to life. The interactive presentation is primarily triggered by the sounds of passing cars. In this instance people did not engage with the art actively to manipulate the light performance, in fact no one was observed as having paid attention to the display of the LED lights. Although, indirectly they were inevitably the source of the interaction and through this implicit interactivity the artwork 
is realised. According to the artworks' introductory statements (Taipower 2013) A power station represents the foundation of technology, therefore by means of employing interactivity as a creative medium to craft this art installation, it highlights the feature of this specific institution, whilst electricity is a necessary source for establishing a social ecology and environment, in an inconspicuous form (as in most cases we obtain it without obstacles), it is intended to symbolize an implicit and inseparable relationship between people, the environment and electric power.

Fast or Slow, on the other hand, responds to the movement of passersby as a direct source for the interactive performance so as to display its stated artistic intention of evoking a delicate interrelationship between people, environments, and social systems. However, within this interactive performance, a spontaneous and conscious engagement from participants is believed to be essential. According to Koichiro Miura (the artist who created this artwork):

\footnotetext{
"the sensor operates the LED panels located 1.2 meters away so that the passer-by sees the panels in front of him turn on, which intends to arouse the emotion of chasing the light (task, duty, achievement). When he walks in the opposite direction, the panels behind him turn on to arouse the emotion of being chased by the light. The LED light turns off in about two seconds indicating the frequent changes of the social system"(Miura 2011) .
}

Nevertheless during the several field observations, we neither observed anyone stop to watch the movement of the LED bars nor to chase the LED lights.

The Flower Clusters exhibits a vivid kaleidoscope-like performance autonomously. By incorporating people into its interactive display, the intention is to manifest a creative notion of a pleasant and diverse cultural landscape. In order to achieve this objective, the promotion of a curious attitude is crucial, so as to motivate the audience to seek the image capturing device. The main flower sculpture is hung beneath the ceiling, a prominent location, where people often gather, passing and lingering, and where many pause to wait for their relatives or friends coming through the arrivals gate. Due to these more contemplative advantages, we observed that people were often attracted by the scale and floral design of the artwork, and in most cases, due to its random changing images and colorful patterns, but not by its interactive mechanism. We conducted several unstructured interviews with people who had stopped and watched the flowing patterns on the petal-like screens, however none of them had noticed that the artwork was in fact an interactive installation. 


\section{Findings}

During the field observations of the three artworks and their potential audiences, not a single individual was observed as having spontaneously sought or attempted to discern the input devices to manipulate the output effects of the interactive presentations, neither had any individual played with the displays of the artworks. In comparison with the presentation of the artworks, the artists' statement for the artworks and activities of the surrounding area, as well as the behaviors of people towards the three selected artworks at the scenes, we identified that the absence of awareness and direct participation can dramatically affect the realization of the stated intent, not only based on different artworks, but in particular their nuances and how the interactivity was incorporated into the creation and realization of the artworks.

In contrast to the original stated artistic intent as well as the outcomes of the field observations, we conclude that people in Between Moving and Still were the indirect but necessary source for the interactive display, as the surrounding sounds were inadvertently produced by them, consequently triggering the diverse LED light performances. Although people did not get out of their cars to make sounds in order to directly influence the LED light display, their daily activities were the key attribute for arousing aesthetic change, which is crucial in order to engage the mediating factor of interactivity. The interactive mechanisms of this artwork are similar to those of Listening Post: where Listening Post obtains its source of interactivity through the internet, Between Moving and Still relies on surrounding noise levels - both to engage specific effects/associations, and to visualize the interrelationship between people and environment. Moreover they not only embody the features of interactive art but also the stated artistic intent as well as further unexpected or unpremeditated associations that invite contemplation and aesthetic engagement.

According to the artist who created Fast or Slow, a physical and conscious intervention from people is an essential element in order to manifest aesthetic interaction of this artwork (Miura 2011). However, perhaps due to the ubiquitous nature of computing media in such public contexts, and the function of the space as fundamentally a corridor connecting to a transport hub, people in the space behaved indifferently to the display of the LED light bars. In general they passed through the area without pausing or even glancing at the artwork. Thus in comparison with the artist's statement, although their movements and activities within the space were registered as an indispensible component of the interactive display, without conscious participation the artwork may not have fully realised.

Similarly, in The Flower Clusters, a spontaneous intervention from people is also vital in order to fully unfold the aesthetic qualities of this interactive installation. Through nonparticipant, participant observation and unstructured interviews with people on site, we 
discovered that many people were attracted to the bright and vibrant images, and many took pictures of the flower sculpture. Apparently, their attention had been caught, which is important if "aesthetic interaction aims for creating involvement experience" (Petersen et al 2004). According to Müller et al (2010) attracting attention is the first threshold in terms of constructing a successful interactive experience in a public context, followed by raising curiosity and eliciting motivation so as to sustain a long term engagement. However, none of the observers and interviewees had discerned the interactive feature of this artwork. We ascribe two major factors that led to their lack of awareness regarding the interactivity of this art installation; firstly the display of people's images only appears occasionally (and only for a few minutes on each display), secondly the image input console is fitted at a distance from the flower installation, and also the design of the input console is stylistically unrelated to the output component. Based on the above issues, we conclude that the intended interactive element of this artwork was not completed, although people's attentions were held for subsidiary reasons.

\section{Summary}

Although unwittingly, people were the key contributor for triggering aesthetic interactions in all three interactive artworks, nevertheless the outcomes were very different in each individual piece. Interactivity was employed in Between Moving and Still and enabled the LED strip (Figures 1-1 -1-3) to respond to the fluctuating auditory environment, including people, though rarely if ever via direct involvement. Through this implicit interaction, the intention of the work was to a very large degree still available. In the cases of both Fast or Slow and The Flower Clusters the embodiment of aesthetic interaction in these two art installations, heightening people's conscious and physical engagement. Nevertheless as previously mentioned, when art enters the public sphere it encounters various challenges, among which involuntary audiences are perhaps one of the most significant. The former artwork (Fast or Slow) appeared to be unattractive to people which may be attributed to the fact that it has been positioned in a thoroughfare and with no clear incentive to people to initiate engagement with the interactive elements. The latter art installation (the Flower Clusters) overcame the first threshold and successfully caught people's attention (Müller et al 2010), however it appeared incapable of directing audience members to appreciate/experience its interactive characteristics. Thus the creative/interactive potential of both Fast or Slow and The Flower Clusters are, to some degree, not full realised. This was perhaps due to the artworks having been created with the primary intent of arousing direct interactivity with people; the widespread notion of interactivity in interactive art, a direct and conscious interaction with people is indispensible, otherwise the purpose of interactive art may not be regarded as having been fully engaged. However, if one relinquishes this inflexible expectation and applies an alternative interpretation of interactivity as a creative medium, 
then the possibilities of art can be broadened, for instance in the artworks Between Moving and Still and Listening Post. By contrasting the stated intent of the artists, to observations and to literature reviews, the concepts of Ambient Interaction and Interactivity as creative medium have emerged. Although these concepts may help to expand the creative scope of artists and art practitioners, we wish to emphasise that the comparative findings are by no means the sole determinant of the artwork's eventual success.

\section{Conclusions}

It is evident that interaction has a key role in the discussed art genre, however it may not be necessary to exclusively interact directly with people in order to realise the artwork. Instead of claiming a new concept of Ambient Interaction, it is perhaps more appropriate to say that this article proposes an expanded conceptualization of interactivity as a creative medium. We value and appreciate artworks in light of their capabilities when promoting interactivity. Nevertheless, we advocate that the interactivity in the realm of interactive art can be presented with more dynamic approaches, which do not necessarily need to be confined to direct action between artworks and people, but can be more flexible and broadly involved with other environmental attributes for the realization of the potential of art. Rather than insist on the generation of ideas as a result of direct interaction between artworks and people, we suggest that during the design process, artists and art practitioners might benefit from taking into consideration not only whom, but more broadly what their artworks will encounter and interact with in the assigned space. In either case, interactivity has to be appropriately employed as a creative medium in order to display its full potential. In addition, although the concept of Ambient Interaction initially emerged through field observations of interactions between the interactive artworks and people in public spaces, it can also be applied to work which is intended to be displayed in other exhibition contexts. In the commissioning and production of interactive artworks there is much to be gained by cultivating a flexible point of view, which regards interactivity as a creative medium, which can be employed to create artworks that are not restricted to a single channel of human interaction, but that can either adapt to wider environmental causal influences or respond more generally to ambient conditions. 


\section{References}

Ascott, R., ed. 1999. Reframing Consciousness: Art, Mind and Technology. Exeter, UK: Intellect.

Bialoskorski, L. S., Westerink, J. H. and Van den Broek, E. L. 2010. "Experiencing affective interactive art". International Journal of Arts and Technology 3(4): 341-356.

Bilda, Z., Bowman, C. and Edmonds, E. 2008. "Experience evaluation of interactive art: study of GEO landscapes". In Proceedings of the 5th Australasian Conference on Interactive Entertainment. Brisbane, Queensland, Australia: ACM.

Boros, D. 2012. Creative Rebellion for the Twenty-First Century: The Importance of Public and Interactive Art to Political Life in America, New York, USA: Palgrave Macmillan.

Brignull, H. and Rogers, Y. 2003. "Enticing people to interact with large public displays in public spaces". Human-Computer Interaction - Interact'03. Zurich, Switzerland: IOS Press.

Ciolfi, L., Cooke, M., Bertelsen, O. and Bannon, L. 2005. "Understanding and Co-Designing for Aesthetic Experience”. Workshop. Edinburgh, UK: HCI 2005.

Dewey, J. 2005. Art As Experience. paperback ed. New York: Penguin Group.

Dezeuze, A. ed. 2010. The 'Do-it-Yourself' Artwork: Participation from Fluxus to New Media. Manchester and New York: Manchester University Press.

Downes, E. J. and McMillan, S. J. 2000. "Defining interactivity a qualitative identification of key dimensions". New Media \& Society 2(2): 157-179.

Edmonds, E., Everitt, D., Macaulay, M. and Turner, G. 2004. "On physiological computing with an application in interactive art". Interacting with computers 16(5): 897-915.

Edmonds, E. 2010. "The art of interaction”. Digital Creativity 21(4): 257-264.

Edmonds, E. 2011. “Art, interaction and engagement". In proceeding of Information Visualisation (IV), 451-456. Sydney, Australia: IEEE.

Hansen, M. and Rubin, B. 2002. "Listening post: Giving voice to online communication". In Proceedings of the 2002 International Conference on Auditory Display. Kyoto, Japan: ICAD.

Hein, H. 1996. "What is public art?: Time, place, and meaning". The Journal of Aesthetics and Art Criticism 54(1): 1-7.

Heinrich, F. 2011. "Investigating Interactive Beauty - A research-art installation”. ISEA2011, Istanbul. Accessed 17 April 2013. http://isea2011.sabanciuniv.edu/paper/investigating-interactive-beauty-\%E2\%80\%93-re search-art-installation.

Huang, E. M., Koster, A. and Borchers, J. 2008. “Overcoming assumptions and uncovering practices: When does the public really look at public displays?". In Pervasive Computing 228-243. Berlin Heidelberg: Springer. 
Huhtamo, E. 2004. Trouble at the Interface, or the Identity Crisis of Interactive Ar. Media Art Histories Archive. Accessed 7 June 2009. http://193.171.60. 44/dspace/bitstream/10002/299/1/Huhtamo.pdf.

Horowitz, G. M. 1996. "Public art/public space: The spectacle of the Tilted Arc Controversy". The Journal of Aesthetics And Art Criticism 54(1): 8-14.

Höök, K., Sengers, P. and Andersson, G. 2003. "Sense and sensibility: evaluation and interactive art". In Proceedings of the SIGCHI conference on Human factors in computing systems, 241-248. Florida, USA: ACM.

JiunsView. 2013. "The Flower Clusters”. Accessed 17 April 2013. http://www.jiunsoutlook.idv.tw/computer-animation/theflowerclusters.jpg

Kim, K. N. and Kim, T. 2012. "Utilization of material-focused paintings in interactive art through the analysis of immersive elements". Digital Creativity 23(3-4): 278-290.

Knickmeyer, R. L. and Mateas, M. 2005. Preliminary evaluation of the interactive drama facade. In CHI'05 Extended Abstracts on Human Factors in Computing Systems, 1549-1552. ACM.

Knight, C. 2008. Public Art: Theory, Practice and Populism. USA, UK and Australia: Blackwell.

Krueger, M. W. 1977. "Responsive environments". In Proceedings of the national computer conference, 423-433. New York, USA: ACM.

Kwon, M. 2004. One place after another: Site-specific art and locational identity. Cambridge, Massachusetts and London, England: MIT press.

Larsson, A. O. 2011. "Interactive to me-interactive to you? A study of use and appreciation of interactivity on Swedish newspaper websites". new media \& society 13(7):1180-1197.

Malloy, J., ed. 2003. Women, art, and technology. USA: The MIT Press.

Manovich, L. 2001. "Post-media Aesthetics Medium in Crisis". Accessed 17 April 2013. http://www.alice.id.tue.nl/references/manovich-2005.pdf

Miura, K. 2011. "Fast and Slow". Accessed 17 April 2013. http://www.miuraarchitecture.com/0807fast_slow/0807fast_slow.htm

Müller, L., Edmonds, E. and Connell, M. 2006. "Living laboratories for interactive art”. CoDesign 2(4): 195-207.

Müller, J., Alt, F., Michelis, D. and Schmidt, A. 2010. "Requirements and design space for interactive public displays". In Proceedings of the international conference on Multimedia, 1285-1294. Firenze, Italy: ACM.

Paulos, E. 2007. "HCI Cannot Be Used to Evaluate Art". Human-Computer Interaction Institute, Carnegie Mellon University. Accessed 17 April 2013. http://repository.cmu.edu/cgi/viewcontent.cgi?article=1208\&context=hcii 
Petersen, M. G., Iversen, O. S., Krogh, P. G. and Ludvigsen, M. 2004. "Aesthetic Interaction: a pragmatist's aesthetics of interactive systems". InProceedings of the 5th conference on Designing interactive systems: processes, practices, methods, and techniques, 269-276. New York, USA: ACM.

Popper, F. 2007. From technological to virtual art, Cambridge, Massachusetts and London, England: MIT Press.

Rogala, M. 2005. "Toward a theory of interactive art experience". In Proceedings of the 3rd International symposium of interactive media design, 5-7. Istanbul, Turkey: ISIMD.

Rokeby, D. 1995. "Transforming Mirrors: Subjectivity and Control in Interactive Media". Critical Issues in Electronic Media. Simon, P., ed:133-158. New York: State University of New York Press.

Selwood, S. 1995. The Benefits of Public Art: The polemics of permanent art in public places. London, UK: Policy Studies Institute.

Sims, R. 1997. "Interactivity: A forgotten art?." Computers in Human Behavior,13(2): 157-180.

Sundar, S. S., Xu, Q. and Bellur, S. 2010. "Designing interactivity in media interfaces: A communications perspective". In Proceedings of the 28th international conference on Human factors in computing systems, 2247-2256. Atlanta, USA: ACM.

Taipower. 2013. “Cover Story”. Accessed 17 April 2013. http://info.taipower.com.tw/TaipowerWeb//upload/files/29/TP580_p6-15.pdf

Taiwan Public Art. 2012. "Collection”. Taiwan Ministry of Culture. Accessed 17 April 2013. http://publicart.moc.gov.tw/eng/search/content.php?id=2434

Tikka, H., Viña, S., Jacucci, G. and Korpilahti, T. 2011. "Provoking the city-touch installations for urban space". Digital Creativity 22(3): 200-214. 


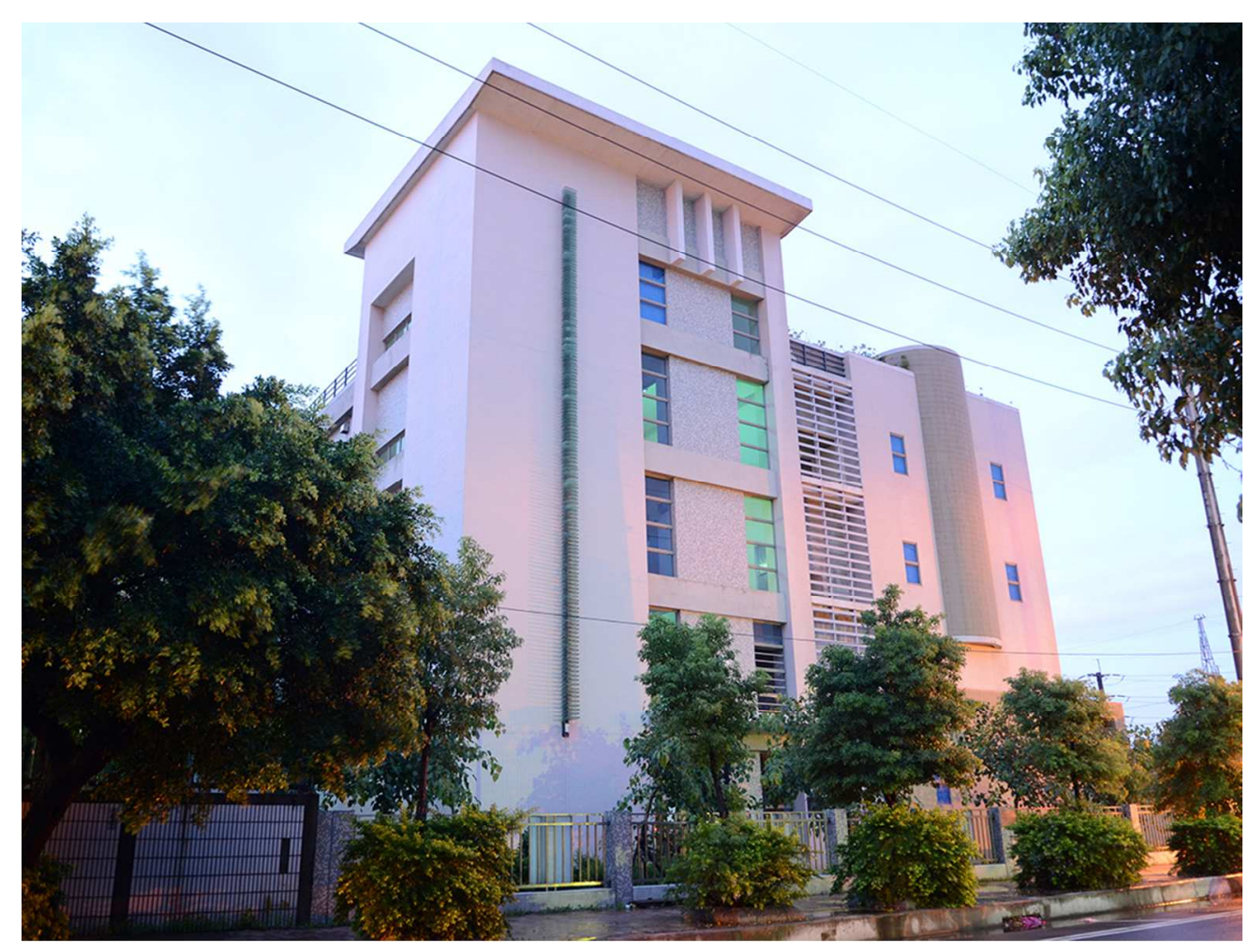

Between Moving and Still 238x179mm (120 x 120 DPI) 


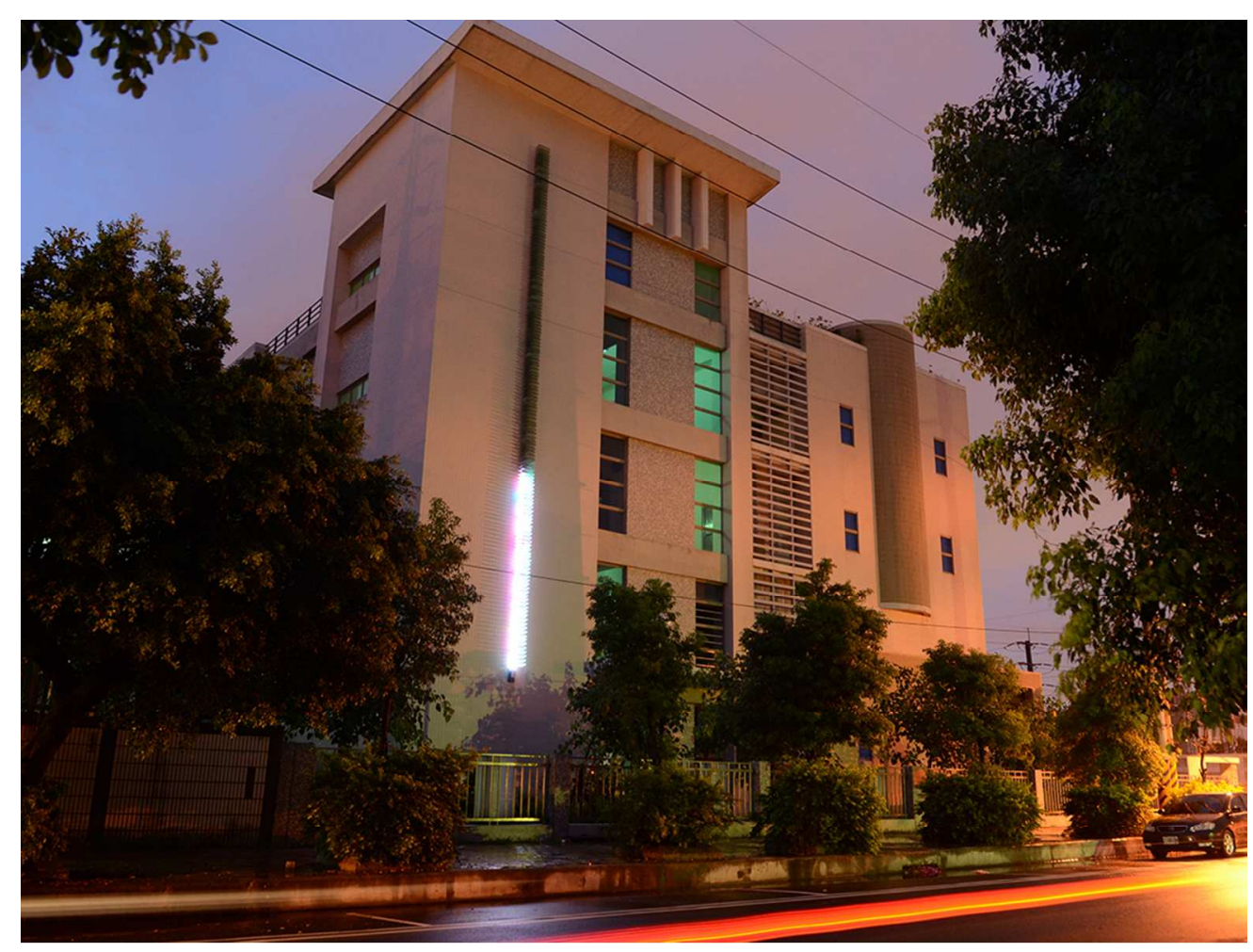

Between Moving and Still $238 \times 179 m m(120 \times 120$ DPI $)$ 


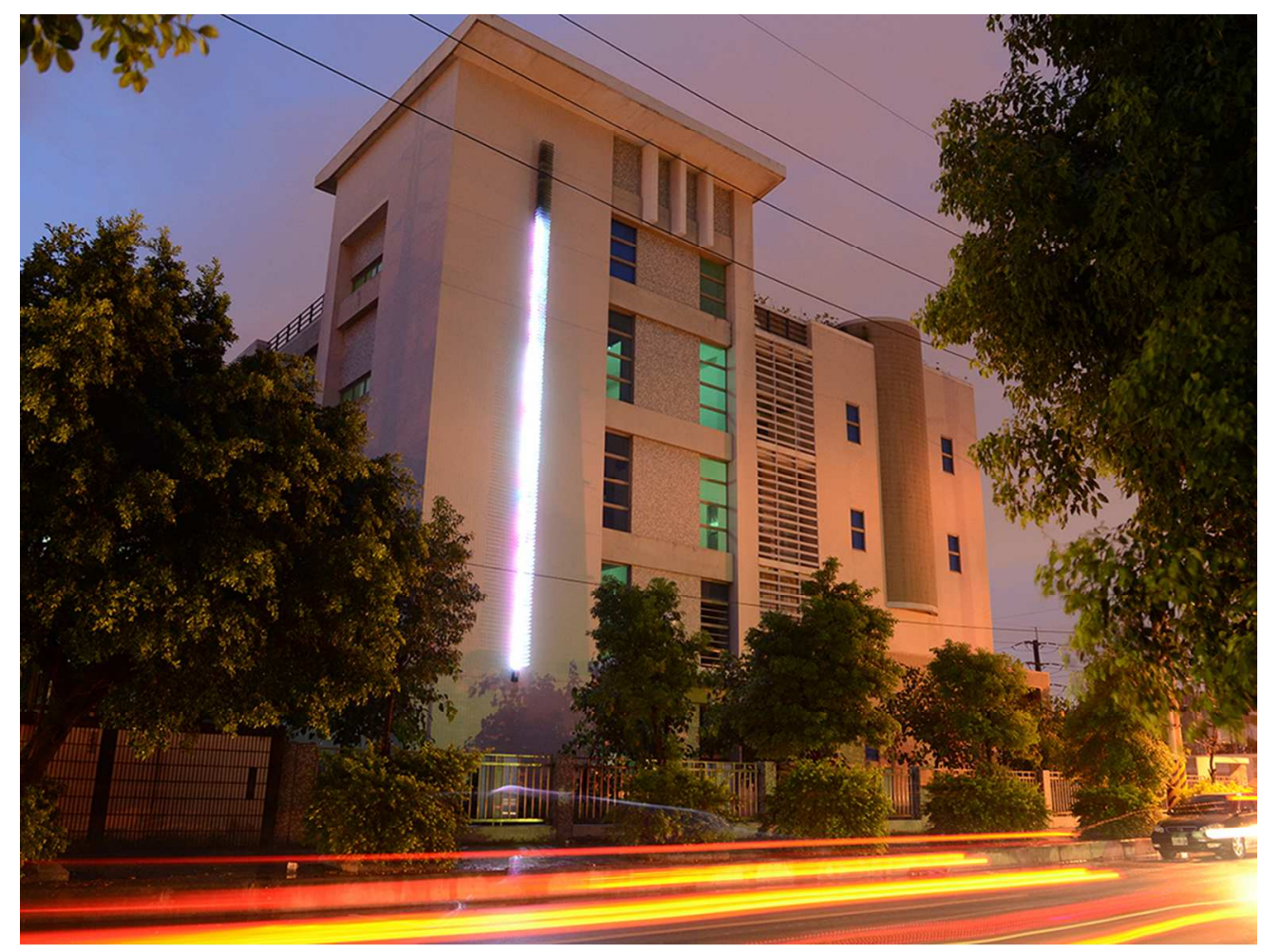

Between Moving and Still 238x179mm (120 x 120 DPI) 


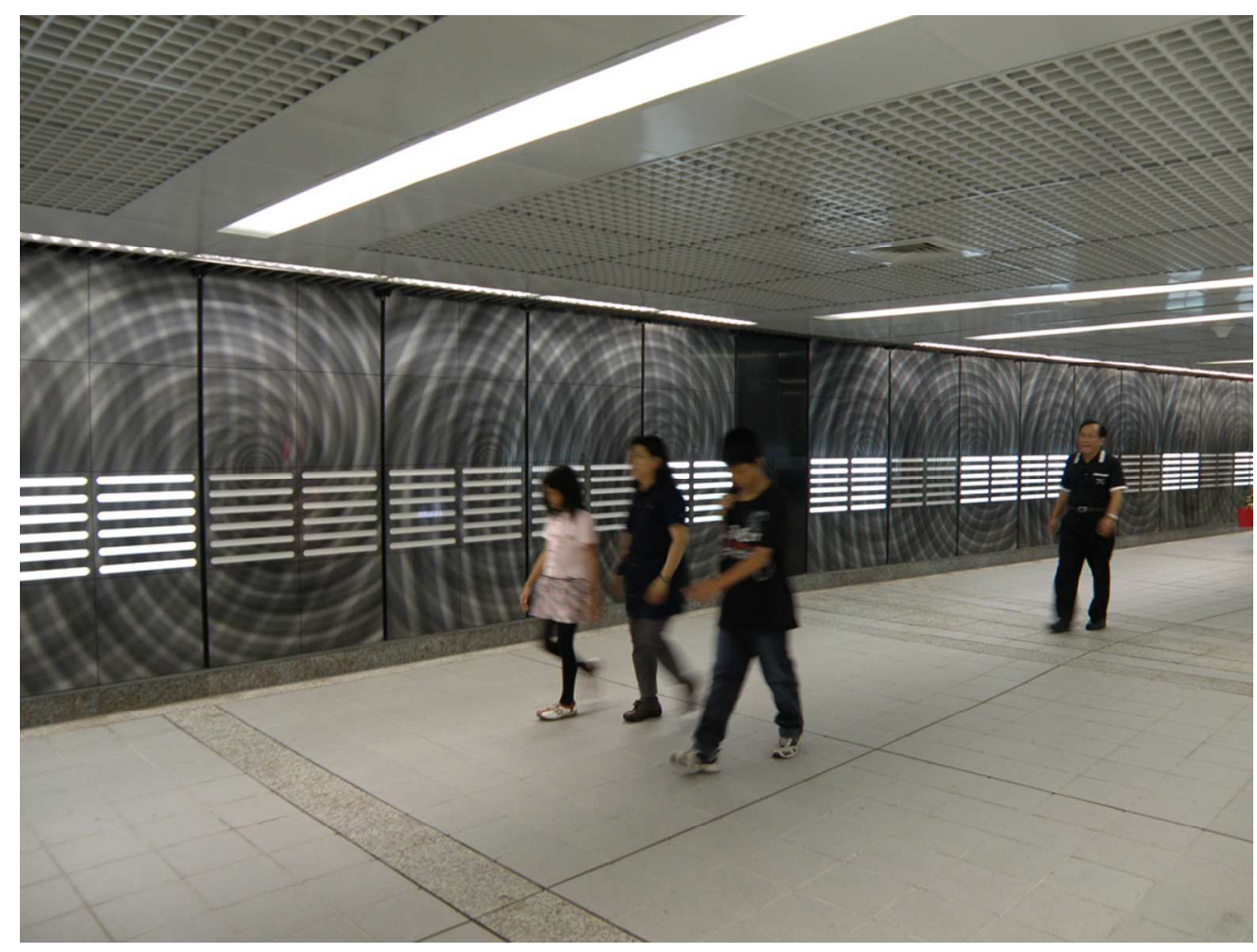

Fast and Slow

$238 \times 179 \mathrm{~mm}(120 \times 120 \mathrm{DPI})$ 


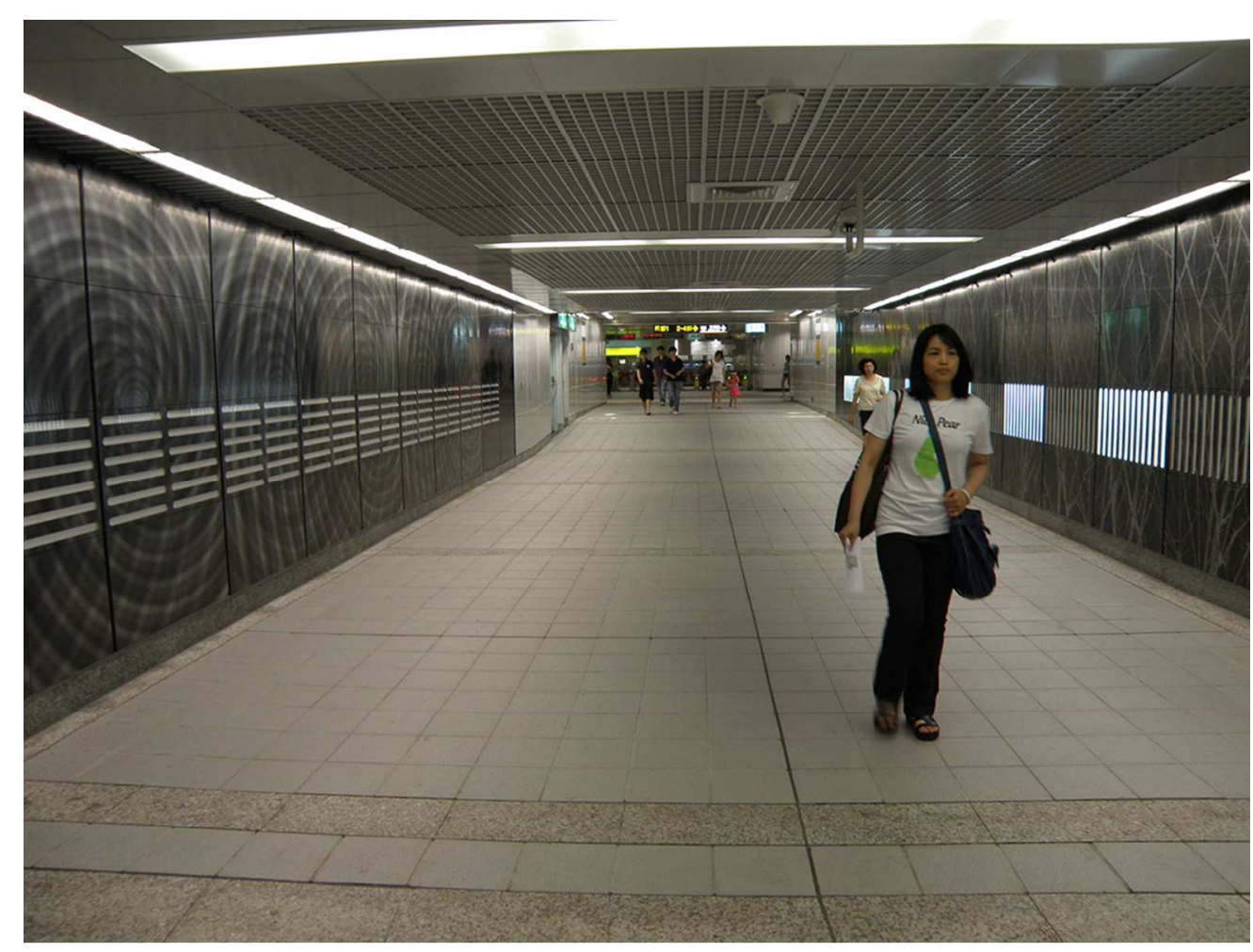

Fast and Slow

$238 \times 179 \mathrm{~mm}(120 \times 120 \mathrm{DPI})$ 


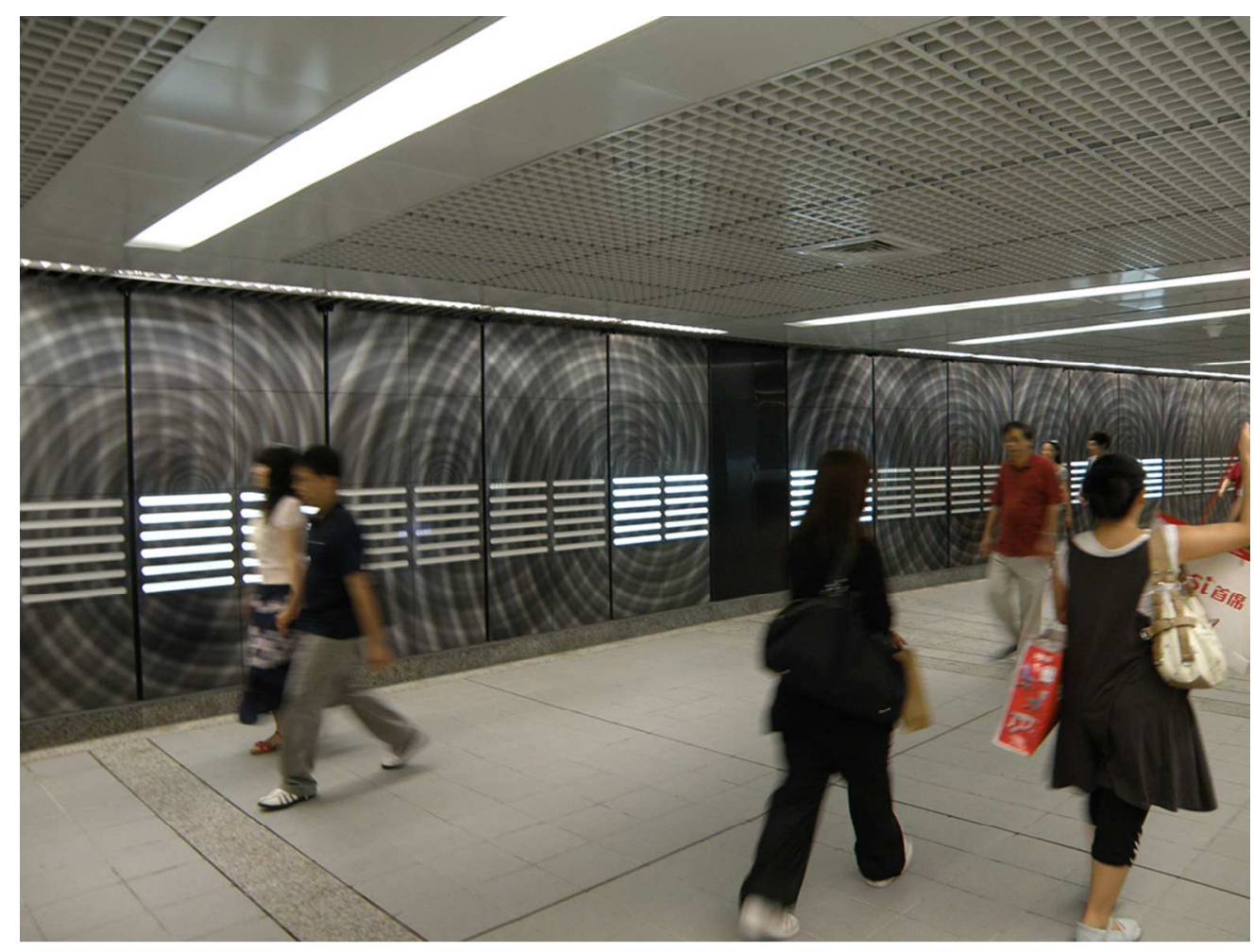

Fast and Slow

$238 \times 179 \mathrm{~mm}(120 \times 120 \mathrm{DPI})$ 


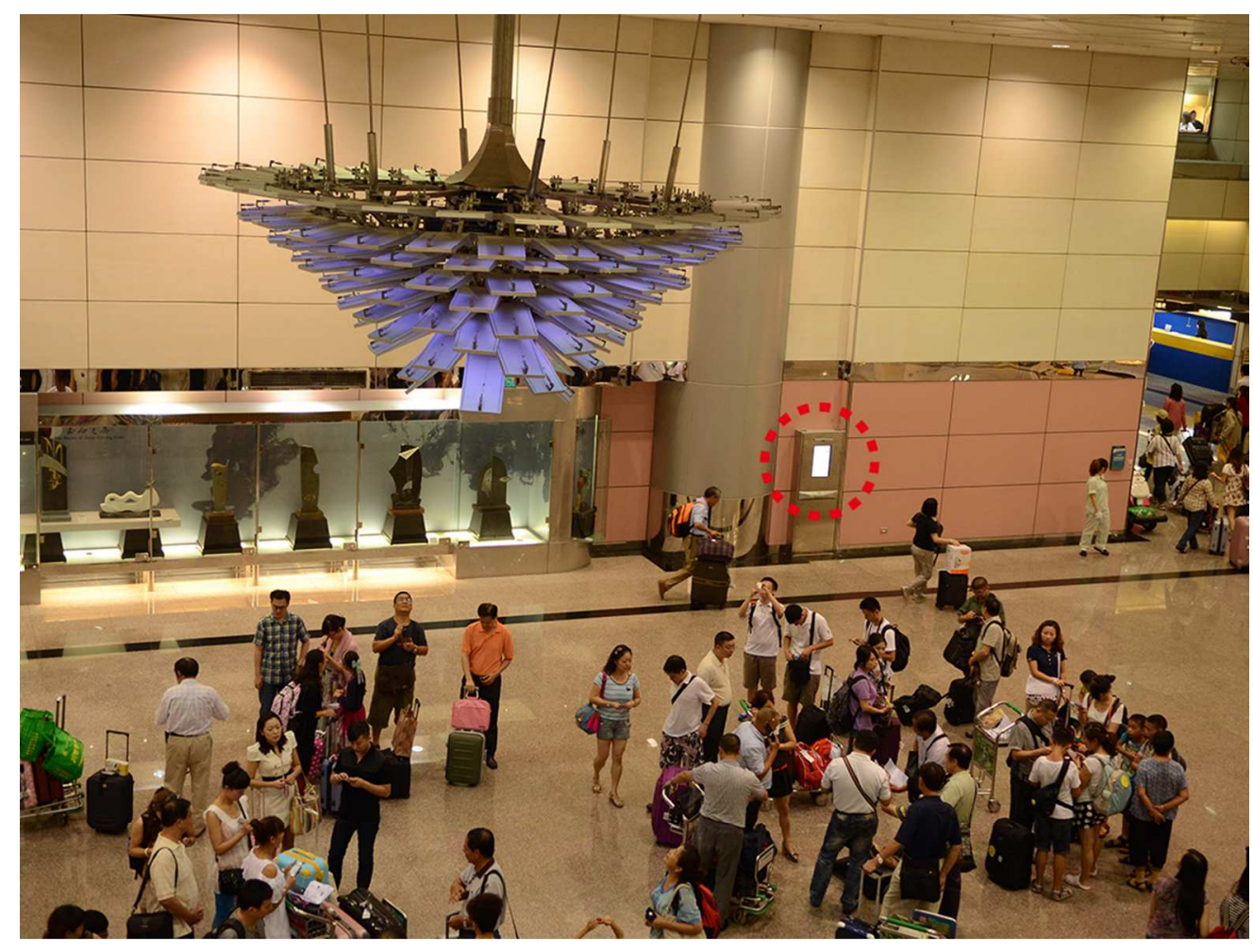

The Flower Clusters $238 \times 179 \mathrm{~mm}(120 \times 120$ DPI $)$ 


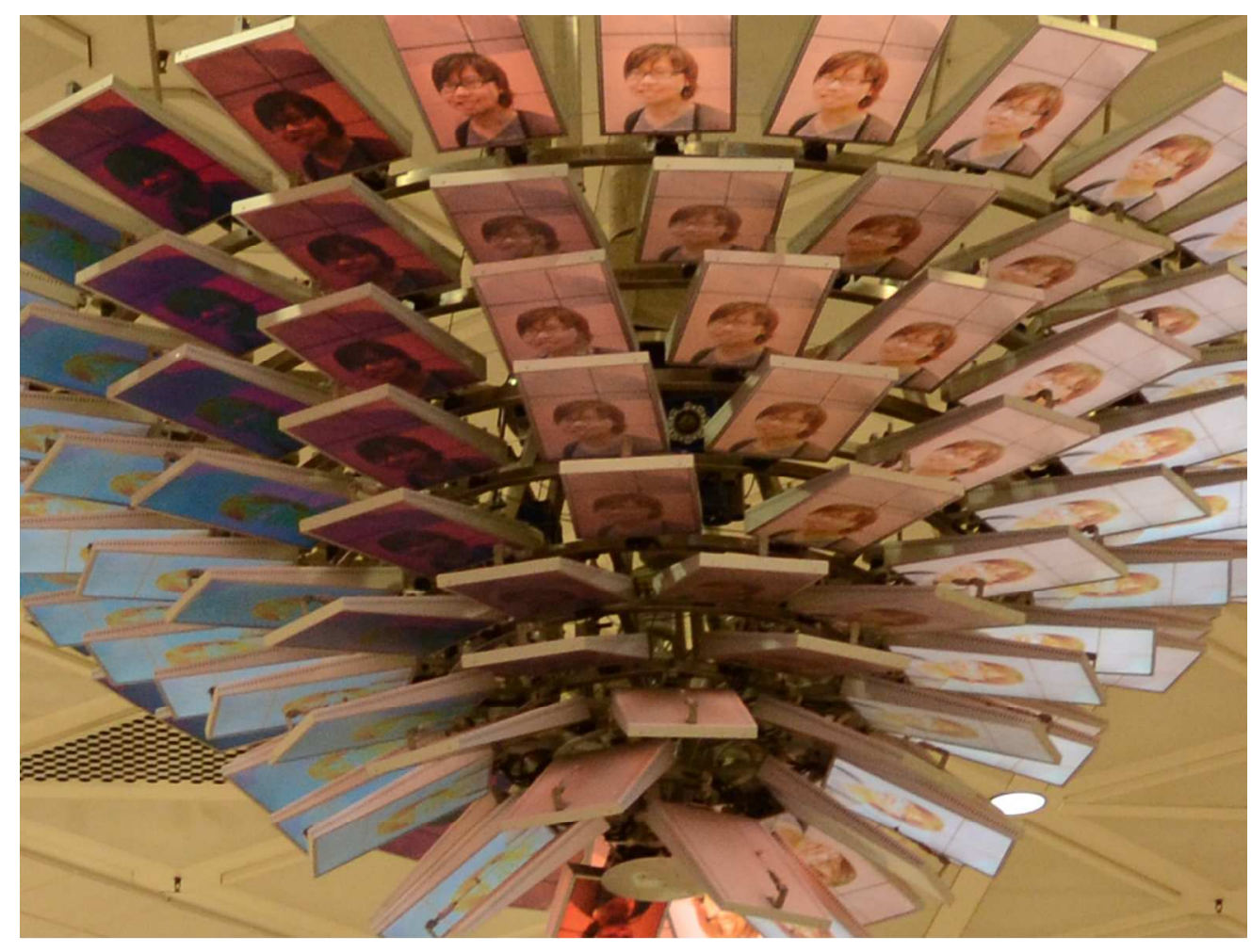

The Flower Clusters $238 \times 179 \mathrm{~mm}(120 \times 120 \mathrm{DPI})$ 


\section{Page 25 of 25}

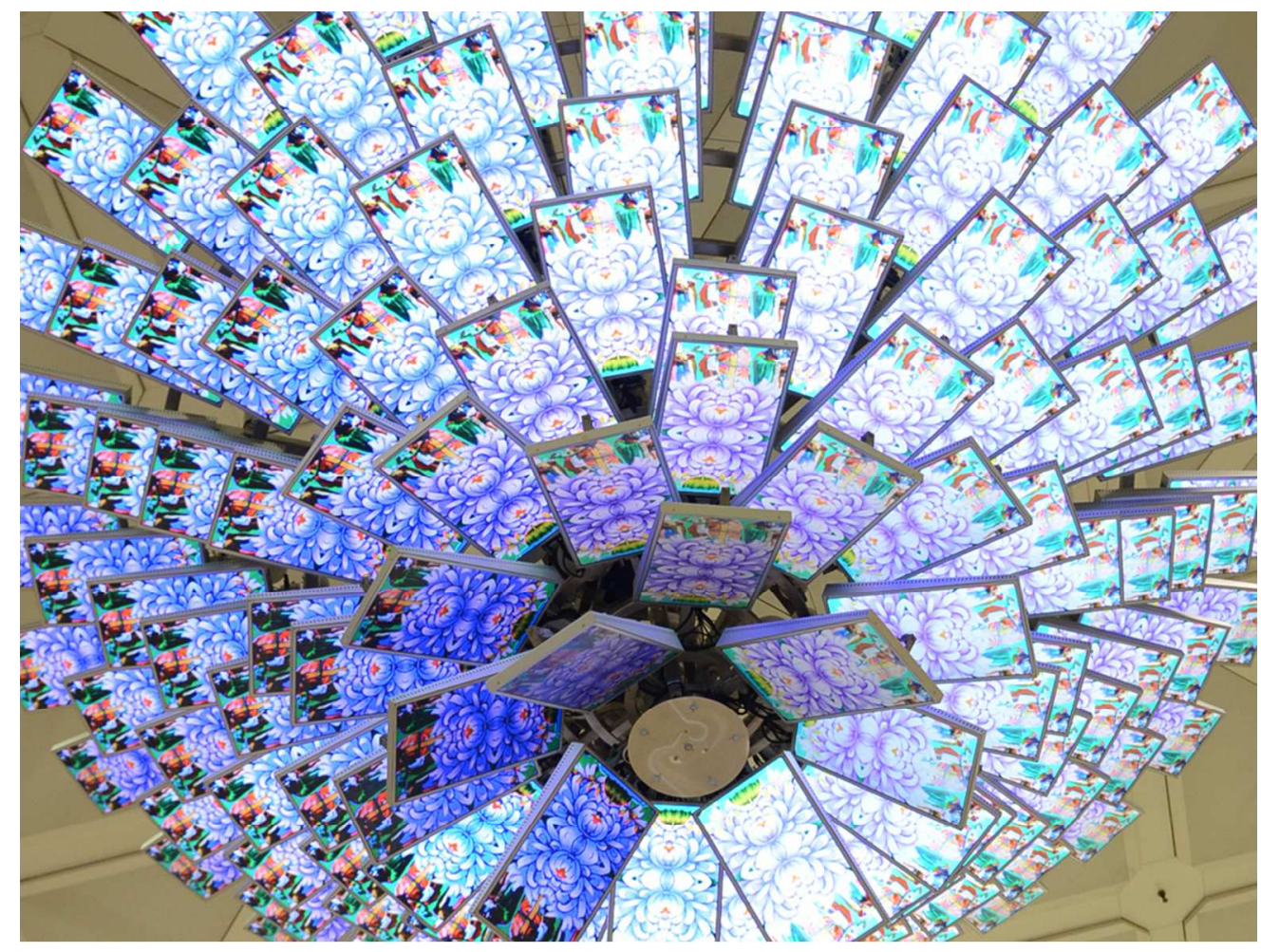

The Flower Clusters $238 \times 179 \mathrm{~mm}(120 \times 120 \mathrm{DPI})$ 